Itinéraires Itinéraires

Littérature, textes, cultures

\title{
Le pastiche du roman-feuilleton dans Le Voleur de Georges Darien et ses adaptations
}

The Pastiche of the Serialized Novel in The Thief by Georges Darien and its Adaptations

\section{Aurélien Lorig}

\section{OpenEdition}

\section{Journals}

Édition électronique

URL : http://journals.openedition.org/itineraires/7506

DOI : $10.4000 /$ itineraires.7506

ISSN : 2427-920X

\section{Éditeur}

Pléiade

\section{Référence électronique}

Aurélien Lorig, « Le pastiche du roman-feuilleton dans Le Voleur de Georges Darien et ses

adaptations », Itinéraires [En ligne], 2020-1 | 2020, mis en ligne le 02 octobre 2020, consulté le 14

novembre 2020. URL : http://journals.openedition.org/itineraires/7506 ; DOI : https://doi.org/10.4000/ itineraires.7506

Ce document a été généré automatiquement le 14 novembre 2020.

\section{(c) (i) () $\Theta$}

Itinéraires est mis à disposition selon les termes de la licence Creative Commons Attribution - Pas d'Utilisation Commerciale - Pas de Modification 4.0 International. 


\title{
Le pastiche du roman-feuilleton dans Le Voleur de Georges Darien et ses adaptations
}

\author{
The Pastiche of the Serialized Novel in The Thief by Georges Darien and its \\ Adaptations
}

Aurélien Lorig

Georges Darien est un écrivain contestataire de la Belle Époque pour qui l'art de la composition fait partie intégrante du projet romanesque qu'il présente dans sa correspondance en 1889. En effet, durant cette année, l'écrivain pose les premiers jalons d'une réflexion concernant l'éthique et l'esthétique de son œuvre future. Outre l'influence remarquable du roman de mœurs réaliste et naturaliste au $\mathrm{XIX}^{\mathrm{e}}$ siècle, Darien défend l'idée d'une série de romans " pétards " qui alterneraient avec des récits jugés « inoffensifs » (Stock 1935 : 74). D'un point de vue organisationnel, cette ambition, l'auteur la compare explicitement au réalisme balzacien de la comédie humaine: «Lorsque j'ai écrit Le Voleur, j'avais fait un plan d'une série de romans dont je voulais faire une sorte de nouvelle Comédie humaine. Le premier était Le Voleur [...] 》 (cité dans Auriant 1955 : 28). L'écrivain semble construire l'ensemble de sa démarche autour d'une sorte de roman des origines qui aurait pour titre Le Voleur. En réalité, il s'agit de son troisième roman publié en 1897 après Bas les coeurs! et Biribi.

2 Rédigée pendant l'exil londonien de Darien, cette œuvre de fiction est considérée comme le texte illustrant le mieux les idéaux libertaires de l'écrivain fin-de-siècle. De fait, à travers les aventures de Georges Randal, jeune bourgeois devenu le chantre d'un anarchisme pratique reposant sur la reprise individuelle et la propagande par le fait, Darien construit un ensemble d'aventures réparties en trente chapitres relativement brefs, lesquels, rien que par l'intitulation, annoncent un programme narratif proche du schéma générique du roman-feuilleton. Ce dernier, sans être à proprement parler un genre littéraire puisque le terme renvoie avant tout à un mode de publication dans les journaux de l'époque ${ }^{1}$, fait tout de même écho à une catégorie générique que connaît bien Darien : le roman populaire ${ }^{2}$. Dans l'histoire de ces récits qui apparaissent après la 
Révolution de 1789 avec des auteurs comme Ducray-Duminil, ce qui intéresse Darien c'est la manière dont on conçoit et pratique le genre après 1870 . De fait, ce n'est pas tant la figure du justicier - type Rocambole ${ }^{3}$ - qui intéresse l'écrivain du Voleur, mais plutôt celle de la victime d'un système, là où l'individu ne peut pas librement exister et s'affirmer hors du carcan bourgeois. Ainsi, en toute logique, Le Voleur s'inscrit dans la lignée de héros populaires des années 1880 tels que Roger-la-Honte ${ }^{4}$.

Ce rapprochement du romanesque darienien avec le roman populaire est à mettre en relation avec la stratégie de l'auteur qui n'est pas de reproduire fidèlement les recettes d'une mode littéraire, mais bien de pasticher le genre en question. On touche ici au phénomène que Genette appelle hypertextualité dans son ouvrage Palimpsestes. Le pastiche est un mode d'écriture qui repose sur un modèle de référence que l'on imite selon certains principes (style, idées, genre, etc.) car, comme le souligne Genette, «le pasticheur se saisit d'un style [...] et ce style lui dicte un texte » (Genette $1992: 89$ ). Sans tomber dans une fidélité mimétique, Darien nous sert, en réalité, une fausse monnaie populaire car tantôt il suit les principes de «l'imitation d'un modèle stylistique » (88), tantôt il dépasse le pastiche et gagne même la parodie en relâchant le lien qui unit le modèle au récit ${ }^{5}$. Dès lors, l'idée de pastiche est complexe dans l'écriture du Voleur puisque l'imitation en question est celle de tous les contrastes, faisant même cohabiter le parodiste et le pasticheur, ce qui inscrit la lecture dudit roman dans une transtextualité multidirectionnelle. Et la complexité ne s'arrête pas là puisque la double entrée se lit également dans les choix idéologiques qui sont ceux de Darien. D'un côté, le récit est construit comme une suite qui réactive sans cesse une tension narrative ${ }^{6}$ destinée à déjouer les horizons d'attente du public. Effectivement, dans l'indétermination provisoire qui caractérise le récit à intrigues du Voleur, Darien fait du lecteur un acteur à part entière, lequel doit envisager le texte dans un rapport affectif pour ensuite apporter une réponse interprétative aux questions que soulève le roman. Parmi ces questions, il y a, bien entendu, le traitement du pastiche. De l'autre, le récit n'en demeure pas moins un réservoir d'idées parfaitement ancrées dans les problématiques de l'écriture révoltée au tournant du siècle - de Vallès à Mirbeau en passant par Léon Bloy, ce qui revient à décentrer l'observation de la pratique du pastiche vers d'autres enjeux cette fois idéologiques plutôt que formels et génériques.

Cet ancrage très concret pour le lecteur replace Le Voleur dans la lignée des mystères urbains de l'époque, lesquels, il faut le rappeler, connaissent un essoufflement sérieux au moment où Darien imagine à Londres les aventures littéraires de Georges Randal. Issus du roman feuilleton, les mystères ainsi dénommés par la marque adjectivale articulent le cadre urbain, la représentation du crime et l'exploration de nature sociale ${ }^{7}$. Ces romans tiennent à la fois de la fiction et de l'exposé d'opinion puisque le mystère urbain vient " s'ancrer dans l'époque contemporaine, s'intéresser au quotidien ordinaire de ses personnages et porter les marques intrinsèques de l'actualité " (Horvath 2008: 23). L'approche du mystère urbain par Horvath fait indéniablement écho aux aventures du Voleur, car Darien fait évoluer son héros révolté dans l'univers des villes, lequel offre la possibilité à l'écrivain de mêler intrigues et introspections critiques des différents personnages. Malgré cette double approche susceptible de captiver le lecteur, le récit des aventures de Georges Randal a été goûté par les lettrés de l'époque, mais pas par le public, ce qui, dans une certaine mesure, constitue un paradoxe puisque le principe même du pastiche du roman populaire touche, finalement, une élite littéraire. La réception du texte ne fait que le confirmer puisque $L e$ Voleur est remarqué par Jarry, Rachilde, Darzens, Descaves ou encore Allais qui, dans Le 
Journal du 24 décembre 1897 ajoute à un «Conte de Noël » une dédicace en faveur de Darien, "auteur de cet admirable Voleur qu'on devrait voir entre toutes les mains dignes de ce nom » (Gréau 2002 : 241). Outre ces personnalités, on peut également citer Roland de Marès qui, dans L'Humanité nouvelle s'intéresse au roman en 1899, lequel est loué pour «le plus grand mépris des préjugés sociaux et même des préjugés révolutionnaires ", le « mépris des conventions littéraires » et l'« imagination de grand feuilletoniste» (238) de l'écrivain. Cette reconnaissance du monde littéraire et journalistique tranche avec la réalité du monde éditorial qui rappelle par la voix de Pierre-Victor Stock, éditeur du Voleur de Darien, que sur un plan financier c'est un échec: non seulement Stock perd 2000 francs avec ce livre, mais en plus Darien ne récupère que 386 francs dans une période où, selon son éditeur, «il est presque impossible de vendre un roman » (Stock 1935 : 85). Pourtant, malgré ces obstacles, Le Voleur n'en demeure pas moins un pastiche des récits populaires parus en feuilletons. Ce choix de l'auteur permet de mettre au jour, d'un chapitre à l'autre, un style et une éthique réfractaires.

Dès le paratexte, Darien brise, en effet, les images convenues. Ce qui entoure le texte fonctionne, à bien des égards, comme une manière de le réceptionner et d'y voir une série d'indices en lien étroit avec le pastiche ensuite à l'œuvre dans le roman lui-même. Du discours de l'avant-propos aux titres de chapitres, le romancier édifie un univers romanesque où l'environnement textuel innerve le récit et ses enchaînements. L'intrigue offre, dès les seuils ${ }^{8}$ de l'œuvre, un faisceau d'indices où, malgré le caractère facétieux de l'écrivain, il est aisé d'identifier la présence de l'idéologie du feuilleton: conditions de publication et sérialité ${ }^{9}$; prise en compte du lectorat et de ses goûts ; ressources génériques variées allant du roman policier au roman noir, en passant par le roman d'intrigue sentimentale; techniques romanesques telles que la composition dramatique serrée et fertile en intrigues, péripéties et coups de théâtre ; représentation de types avec des personnages qui perdent en complexité psychologique et qui illustrent une morale toujours conventionnelle. Pour autant, la reprise dans l'avantpropos au Voleur de cette idéologie laisse place à un détournement de la part de Darien qui n'hésite pas, au-delà du pastiche, à parodier le style et l'idée des feuilletons, et ce, à des fins libertaires. Dès lors, Darien joue sur l'ambiguïté du pastiche qui se situe, d'après Genette, «entre la moquerie et la référence admirative " (Genette 1992: 45). Alors que le pastiche consisterait à reproduire la totalité des éléments qui donnent son identité générique à l'œuvre, Darien préfère exploiter la proximité qui existe entre le pastiche et la parodie, la charge et le travestissement, moyen privilégié de mettre en œuvre une satire du roman populaire et des feuilletons en vogue dans la seconde moitié du XIXe siècle. En procédant de la sorte, Darien n'inscrit donc pas servilement son récit dans les pas d'une catégorie romanesque. Au contraire, il crée une sorte d'œuvre hybride où le chapitre en tant qu'élément central contribue à voir autrement un genre journalistique dans une stratégie qui est celle de l'antiroman, à savoir un récit qui, grâce à diverses influences, se situe en marge de toute récupération générique, tant par son organisation générale que par son contenu narratif. Dans cette perspective, il est intéressant d'envisager la lecture du Voleur comme une fiction seuil ${ }^{10}$.

6 L'écriture du Voleur, à l'origine, s'est construite comme le récit d'une transgression, laquelle passe par le jeu quasi systématique avec l'idée même de seuil. Seuil générique d'abord, dans un antiroman qui remet en cause le principe même de la création littéraire par la référence au vol de manuscrit. Seuil paratextuel ensuite, avec un sens romanesque qui se lit avant même d'entrer dans l'intrigue par l'épigraphe bien sûr, 
mais surtout par le travail des titres et de l'organisation en chapitres. Les étapes du parcours de Randal font de cette fiction un moment non seulement d'apprentissage $\grave{a}$ rebours de l'idéologie bourgeoise des origines, mais aussi un roman de l'appropriation, où l'écrivain affirme une volonté libertaire directement inspirée des idées anarchistes au tournant du siècle. Seuil auctorial enfin, car l'auteur marque ici sa présence à travers une figure romanesque qui pourrait avoir la même destinée que l'auteur. Les adaptations au cinéma et en bande dessinée ont pris en compte ces seuils darieniens à travers le séquençage et la planche. Les espaces narratifs du roman sont repensés à la lumière d'une transmédialité qui permet de relire, voire de redécouvrir autrement les aventures de Georges Randal. De fait, le concept de transmédialité aussi appelé narration transmédia consiste à réinvestir un univers narratif original - ici Le Voleur de Darien - à travers différents médias. Le roman apparaît alors comme un contenu adaptable et capable de se décliner dans des supports médiatiques qui apportent, grâce à leurs spécificités d'usage (médium cinématographique et bédéique), un autre regard sur l'intrigue du roman de 1897 qui nous intéresse dans cet article ${ }^{11}$. Cette transmédialité, tout en respectant les grandes lignes directrices de l'intrigue originelle, apporte ainsi par d'autres techniques une dimension nouvelle au texte fictif. De la sorte, ces adaptations du Voleur au $\mathrm{xx}^{\mathrm{e}}$ siècle ont su intégrer avec d'autres contraintes génériques les traits caractéristiques du roman fin-de-siècle faisant de ce texte une œuvre plastique.

\section{Le roman-feuilleton : un cadre idéologique vecteur de romanesque}

7 L'influence du roman-feuilleton dans Le Voleur ne consiste pas simplement à imiter un genre, mais plutôt à en tirer avantage afin de mettre en place un récit d'apprentissage que l'arrière-plan feuilletonesque rend plus attractif pour le lecteur. Ainsi, la présence des caractéristiques du feuilleton sert une entreprise littéraire dressée contre la bourgeoisie et toutes les figures de l'autorité qui incarnent aux yeux du héros corruption et amoralité. Ce sentiment est justement renforcé par le recours aux ficelles d'une pratique littéraire faisant office dans Le Voleur de catalyseur, lequel déclenche une série de repères dont la finalité est avant tout la démolition d'une mythologie familiale bourgeoise. Cette dernière, partisane et dissimulatrice ${ }^{12}$, est démasquée à travers la présence récurrente du roman-feuilleton. Dans Le Voleur, ce dernier obéit à une série d'influences directement liées au cadre idéologique du feuilleton. Selon Lise Queffélec (1989), on peut établir une périodisation en trois temps rendant compte de l'évolution du genre. Ainsi, le roman de Darien pourrait apparaître comme une sorte de triptyque idéologique où la chronologie du roman-feuilleton est toujours là, en filigrane.

De la sorte, le roman-feuilleton romantique - première période (1836-1866) - incarné par des figures littéraires telles qu'Alexandre Dumas, Eugène Sue, Paul Féval ou encore Frédéric Soulié, inspire en partie l'intrigue du Voleur. Les effets dramatiques de ces feuilletons qui ponctuent le récit de rebondissements, de renversements spectaculaires, de passions destructrices, le tout en présence d'un héros parfois marginal, se retrouvent dans les aventures de Randal. Parmi les réalisations en feuilleton de ladite période, les productions mettent en évidence l'aspect protéiforme des écrits du moment. De fait, les feuilletons puisent leurs ressources dans des genres aussi variés 
que le roman criminel $^{13}$ ou le roman horrifiant ${ }^{14}$. Le premier inspire Darien au nom d'un principe simple que l'écrivain adapte au contexte: un crime commis dans des conditions mystérieuses avec focalisation de l'intérêt du lecteur non pas sur le détective mais sur le criminel, ce qui revient à faire de l'aventure un élément plus important que la résolution de l'énigme en jeu. Le second désigne quant à lui toute cette production romanesque de l'horreur qui est associée par les auteurs de l'époque à l'adjectif noir. Ce dernier renvoie de manière figurée à l'entreprise du Voleur puisque la noirceur met en relief bien des aspects omniprésents dans les courts chapitres qui se succèdent : moralité du Mal, souffrance et présence de la mort, désespoir et affliction. L'ensemble offre aux lecteurs de ces romans horrifiants des émotions violentes placées au service de héros réfractaires qui exercent leur individualisme face à la loi sociale, aux figures de l'autorité et de la justice bourgeoise.

Ces caractéristiques, Le Voleur les met en œuvre dès le paratexte. Le discours de l'avantpropos établit directement le principe du coup de théâtre qui fait de ce texte à venir une œuvre moralement problématique : «Le livre qu'on va lire, et que je signe, n'est pas de moi. [...] Je l'ai volé. J'avoue mon crime» (Darien [1897] 1994 : 7). À la lecture de ces mots, le lecteur songe ici bien plus à une pratique littéraire qu'à une simple confession. Darien inscrit son roman dans une époque de circulation et de reprise accrue de la matière romanesque. Dès lors, le dispositif narratif et pragmatique fondé sur le vol use de cette thématique au second degré puisque le narrateur n'aura de cesse dans le récit de jouer les contrastes quant à ce bien dérobé. Tantôt il le voit comme un outil efficace pour exprimer ses convictions, tantôt il l'envisage comme un moyen d'introspection, objet de dérision amère :

[...] - et que faire de ce manuscrit? En vérité, je n'en sais rien. Je ne veux pas l'emporter et je n'ai point le courage de le détruire. Je vais le laisser ici, dans ce sac où sont mes outils, ces ferrailles de cambrioleur qui ne me serviront plus. Oui, je vais le mettre là. On l'utilisera pour allumer le feu. Ou bien - qui sait ? - peut-être qu'un honnête homme d'écrivain, fourvoyé ici par mégarde, le trouvera, l'emportera, le publiera et se fera une réputation avec. (506)

L'allusion de Darien à ce qui s'apparente à du "romanesque généralisé ", au sens où l'entend Marc Angenot dans ses travaux sur le discours social de l'année $1889^{15}$, ne doit pas faire oublier que Le Voleur est une fiction où le narrateur ménage avant toute chose un horizon d'attente digne des trames feuilletonesques qui sont centrées sur la marge et l'effet de surprise. Cet écart est clairement à l'œuvre si l'on prend en compte l'intitulation du dernier chapitre du Voleur : «Conclusion provisoire - comme toutes les conclusions » (499). Cet excipit ouvert ne peut que laisser le public expectatif, puisque le romancier fait allusion à l'idée d'une suite narrative.

Or, en tenant compte des différents seuils de l'œuvre représentés ici par l'avant-propos et l'excipit, le récit vise un tout autre dessein : ruiner toute récupération morale de la fiction qui, de ce point de vue, conforte l'imaginaire du héros solitaire, marginal et justicier à l'œuvre dans les feuilletons de l'époque. Randal, en abandonnant le livre et en ne proposant pas de conclure ses aventures, ouvre un nouveau chapitre, celui d'un individualisme libertaire et d'un vol aboutissant à la révolte emblématique du héros qui abandonne le livre volé tout en évoquant ce qu'il pourra bien devenir entre les mains d'un autre, non sans désenchantement comme l'atteste la formule finale: «Ah! chienne de vie!» (506). Randal est un vrai voleur capable de s'affranchir d'une matérialité qui aurait pu laisser place à une autre série d'aventures anarchistes, un peu 
à la manière du triptyque vallésien qui voit évoluer la figure de Jacques Vingtras de L'Enfant à L'Insurgé en passant par Le Bachelier.

11 À en croire justement Darien lors d'une polémique au sujet de son titre Le Voleur, l'écrivain aurait projeté d'adapter à la scène l'intrigue de Randal ${ }^{16}$. On découvre ce projet en 1906 au cours d'échanges tendus avec Henry Bernstein qui vient d'annoncer sa nouvelle comédie en trois actes intitulée Le Voleur. Darien prétend à cette occasion avoir un droit d'exclusivité sur le substantif faisant office d'intitulé pour Bernstein : «[...] je lui disais, non seulement que j'avais écrit un roman intitulé Le Voleur, mais encore que j'eusse tiré de ce roman une pièce qui sera sans doute représentée avant peu. [...] Au reste, tout en établissant mon droit à me servir, au théâtre, d'un titre, précédemment employé par moi pour un roman [...]» (Darien 1906 : 2). Cette supposée adaptation confirme le potentiel offert par un roman dont l'organisation interne permet d'imaginer une suite au récit originel, lequel fait montre d'une créativité dès les titres des différents chapitres.

L'intitulation feuilletonesque est véritablement pastichée lorsque l'on parcourt la table des matières : «Rencontres heureuses et malheureuses »; "Aventures de deux voleurs, d'un cadavre et d'une jolie femme»; "Combinaisons machiavéliques et leurs résultats »; « On n'échappe pas à son destin ». Selon Pierre Masson (1994 : 518) dans la postface à l'édition du Voleur chez Seuil, le programme annoncé met en relief, du moins en apparence, un côté picaresque. Georges Randal est bien une sorte de picaro, cette figure de l'antihéros vivant en marge des codes d'honneur propres aux classes dominantes de son époque ${ }^{17}$. Néanmoins, il appartient lui-même à la bourgeoisie, classe détenant tous les pouvoirs au tournant du xix ${ }^{e}$ siècle. Dès lors, le personnage de Darien est davantage la figure d'un roman d'apprentissage où la confrontation avec le milieu bourgeois de son enfance le conduit à s'insurger contre les figures de l'autorité et les tenants de l'idéologie.

13 La partie consacrée au récit familial relève de cette idée d'apprentissage à rebours du bourgeoisisme ${ }^{18}$ tant décrié par l'écrivain. Les trois premiers chapitres se concentrent sur les origines du héros: "Aurore»; «Le cœur d'un homme vierge est un vase profond ${ }^{19}$ »; « Les bons comptes font les bons amis ». La métaphore du commencement, loin d'être poétique, met en route la fabrique d'un feuilleton où le récit des origines est placé sous le sceau de l'obligation de procréer: "Mes parents ne peuvent plus faire autrement " (Darien [1897] 1994 : 13). Les souvenirs de l'enfance racontés par le héros sont marqués par l'argent et le point de vue bourgeois sur le monde. Dès lors, la formule tirée du texte de Musset La Coupe et les Lèvres (1831-1833) résonne, au chapitre II, a contrario, comme un moment crépusculaire puisque le personnage prend conscience, après la perte de ses parents, qu'il a été volé par son oncle et qu'aucune autorité ne laisse libre cours à ses désirs. Le récit laisse place à un constat plein d'amertume : «Je sais que je suis volé. Je vois que je suis volé. [...] Je ne serai pas riche ; je serai peut-être un pauvre» (29). Bien plus que la peur d'un déclassement social hypothétique, la citation servant de titre acte la présence d'une flétrissure morale profonde que l'intertexte de Musset met en relief. Cette référence détermine en quelque sorte le destin de voleur du héros, lequel se confirme au chapitre III à travers un dicton accentuant l'injustice originelle. Georges, tout en débattant de la morale, prend conscience de la fourberie de son oncle qui l'a dépouillé de l'héritage qui aurait dû lui revenir de droit. Ainsi, l'organisation interne du roman met en place une intrigue 
qui prend forme au seuil du récit par un ensemble d'intitulés pensés comme autant de vecteurs de sens.

Outre le travail de l'intitulé de chapitre, Darien sait aussi ménager, toujours dans le cadre idéologique du roman-feuilleton romantique, une influence indirecte provenant du roman noir ${ }^{20}$. Ce dernier se caractérise par un univers violent, un regard tragique sur la société et l'expression d'une morale politique et sociale. Parmi les représentants de cette approche du feuilleton, figure Eugène Sue à qui l'on doit en 1842 Les Mystères de Paris. À sa manière, Darien pastiche le genre en question à travers la figure emblématique de l'abbé Lamargelle. Son identité le place en marge d'une société bourgeoise qu'il juge décadente. Outre l'onomastique qui le prédestine à une prêtrise diabolique, le personnage est un orateur prophète qui prédit la fin d'un temps corrompu par des représentants de l'autorité tantôt grotesques tantôt malfaisants. L'apparition du personnage dans les chapitres du Voleur participe de cette influence remarquable du roman noir, un peu à la façon d'un personnage sombre, sorti tout droit des romans de Lewis et Maturin :

Un soir j'étais seul chez moi [...] lorsque j'entendis résonner le marteau de la porte d'entrée. Un instant après, la voix d'Annie protestant contre l'invasion de mon domicile parvint jusqu'à moi et un pas sourd fit craquer les marches de l'escalier. Je me levai du divan sur lequel j'étais étendu lorsque la porte du salon s'ouvrit à moitié; et par là, par l'entrebâillement, je vis passer une tête bronzée et une main qui faisait des gestes. Quelle était cette main? Quelle était cette tête ? [...]. Cette tête et cette main étaient l'inaliénable propriété de l'abbé Lamargelle [me] saluant, avec l'expression énigmatique de sa puissante figure osseuse et olivâtre [...] obscurcie plutôt qu'éclairée par l'éclat sombre des yeux couleur d'ébène. (171-172)

L'arrivée de l'abbé s'effectue à la jonction de deux chapitres. De la sorte, le suspense est maintenu entier comme si nous découvrions un nouvel épisode de roman-feuilleton. Cette disposition accentue la dynamique en même temps qu'elle conforte la portée davantage humoristique que symbolique des titres des chapitres concernés : «L'art de se faire cinquante mille francs de rente sans élever de lapins" et "De quelques quadrupèdes et de certains bipèdes ». Ainsi, le roman se rapproche plus de la parodie que du pastiche. En procédant de la sorte, le lecteur identifie un « contrat de pastiche » (Genette 1992: 50) qui ne laisse aucun doute possible quant à la volonté de placer l'imitation sous l'égide d'un rire de satire. Le titre fonctionne comme un horizon d'attente qu'il faut envisager au second degré.

Pour autant, cette stratégie de Darien est à replacer, plus largement, dans le cadre idéologique d'une seconde périodisation du roman-feuilleton qui va de la fin du Second Empire aux débuts de la III République. Ces années voient le développement considérable de la presse politique et du quotidien populaire. C'est l'époque où Émile Gaboriau envisage le roman-feuilleton comme une intrigue criminelle à caractère judiciaire, lequel caractère est dérivé du roman policier. L'Affaire Lerouge publié en feuilleton dans le journal Le Pays puis dans Le Soleil, est directement inspiré d'une affaire célèbre qui a défrayé la chronique en 1865. La thématique de la justice est au cœur d'un texte qu'il faut plus largement mettre en perspective avec la période qui se situe après 1875 lorsque le roman-feuilleton en vient à consacrer le roman social, à savoir un roman à la trame mélodramatique qui repose sur l'attendrissement et finit par aboutir sur l'émergence d'un roman du sentiment ${ }^{21}$ dont le prototype est La Porteuse de pain de Xavier de Montepin ${ }^{22}$. 
Outre Xavier de Montepin, figure aussi Jules Mary parmi les auteurs de ce roman social. Toutefois, ce sont plutôt des noms comme Michel Zévaco ou Maurice Leblanc que retiendront les lecteurs de ce roman-feuilleton fin-de-siècle. Darien connaît bien cette tendance remarquable du genre à jouer sur l'attente du lecteur autour d'une intrigue tridimensionnelle: judiciaire, sociale et sentimentale. Dans une certaine mesure, Le Voleur pastiche les traits caractéristiques en question. En effet, le personnage de Roger Voisin est directement inspiré du roman-feuilleton de Jules Mary, Roger la Honte, roman dans lequel le personnage éponyme est victime d'une erreur judiciaire. En procédant lui-même à une correction au sujet de son identité : «Permettez-moi seulement une petite rectification; mon nom est bien Roger Voisin mais, d'ordinaire, on m'appelle Roger la Honte" (Darien [1897] 1994: 110), Darien recourt à un intertexte afin de construire le portrait d'un héros typique du genre, lequel devient un compagnon de vol pour Randal.

Ensemble, les deux jeunes hommes se décrètent voleurs de la bourgeoisie et entendent récupérer à la classe dominante du moment ce qu'elle leur aurait volé de manière plus symbolique que matérielle : leur jeunesse et leur individualisme. De fait, Roger aspire à être un artiste alors même que le monde dans lequel il vit condamne cette vocation. Hormis ce mépris, Roger est aussi face à la nécessité d'avoir de l'argent afin de réaliser son rêve artistique, lequel est tourné vers la ville de Venise. De son côté, Georges pratique le vol selon un schéma purement antibourgeois puisque ses motivations sont familiales, voire intimes lorsqu'il s'agit de son amour impossible pour Charlotte, la fille de son oncle Urbain Randal.

19 Toutefois, ce qu'ils considèrent l'un et l'autre comme des injustices les conduit paradoxalement à être au cœur d'erreurs judiciaires. Randal par exemple finit par devenir un justicier criminel aux chapitres XIX et XX respectivement intitulés «Événements complètement inattendus " et "Où l'on voit qu'il est souvent difficile de tenir sa promesse ». Ces chapitres mettent en place dès le titre un programme narratif digne du roman-feuilleton à la manière de Jules Mary. Georges est confronté à la réalité du cœur puisqu'il se découvre père d'une petite Hélène qu'il a eue avec Charlotte. Cette enfant souffre d'une méningite. À l'intrigue sentimentale se surimpose un récit policier, puisque le héros décide de dérober l'argent de Paternoster afin de pouvoir payer le docteur Scoundrel ${ }^{23}$ pour sa fille agonisante. Toutefois, rien ne se passe comme prévu puisque la suite du récit est une succession de coups de théâtre annoncés par la rhétorique des titres où l'adverbe vient renforcer le caractère feuilletonesque du Voleur : expression d'un degré extrême et d'une fréquence. Conjointement, ces emplois dynamisent le récit au seuil du chapitre. Le déroulé ne fait que le confirmer si l'on établit les péripéties à l'œuvre : chute malencontreuse de Paternoster lorsque Georges lui arrache sa mallette ; mort du faussaire à la suite de sa blessure à la tête ; culpabilité de Georges apprenant la nouvelle par l'abbé Lamargelle qui lui indique qu'un homme a été arrêté pour ce crime, sans avouer, et pour cause; enfin, délitement du couple Charlotte-Georges après la mort d'Hélène qui n'a pas pu être sauvée. Dès lors, le récit nous amène à une véritable intrigue de roman-feuilleton en révélant, en même temps que le pastiche d'un genre, les tourments d'un héros qui fait l'amère expérience d'une destinée bourgeoise lui collant à la peau telle la « casaque » (Darien [1890] 2002:9) de forçat de Jean Froissard ${ }^{24}$. De la sorte, le récit darienien est sous l'influence d'une veine feuilletonesque à rebours de la morale, avec pour dessein de mettre au jour un individualisme en quête de repères loin des figures instituées de l'autorité. En mettant 
en œuvre une dimension moralisante du roman-feuilleton volontairement détournée par Darien, l'auteur rompt avec les vertus morales défendues par des feuilletonistes comme Eugène Sue. En effet, les récits multiples visent à offrir au lecteur un suspense placé au service de la réflexion morale sur les agissements des personnages. Darien ébranle ce trait caractéristique du feuilleton pour faire triompher un Mal dont la nature provient directement de la société fin-de-siècle corrompue. Cette altération laisse apparaître la marginalité d'un roman qui s'inscrit seulement de manière partielle du côté des productions romanesques du XIX siècle. Pour autant, ce pastiche qui vire au détournement constitue tout l'intérêt d'un récit qui surprend et connaît des adaptations pour le moins intéressantes à considérer.

\section{Le Voleur au cinéma : du cadre idéologique à l'effraction générique?}

Cette capacité de Darien à faire rebondir constamment un récit en exploitant les ressorts du roman-feuilleton, d'aucuns dans les différentes adaptations du Voleur parviennent à la construire de manière originale. Du chapitre à la séquence filmique, le cas du Voleur adapté par Louis Malle en 1967 repense l'ordonnancement du roman aussi bien dans ses seuils que dans ses contenus narratifs. Le film met en œuvre l'image d'une figure effractionnaire tant d'un point de vue éthique qu'esthétique.

L'adaptation filmique du texte de 1897 reprend d'une autre manière le programme narratif à l'œuvre dans les trente chapitres de la fiction ${ }^{25}$. D'un point de vue esthétique, la réalisation en couleurs transpose au cinéma un certain nombre d'éléments romanesques. Parmi eux, on note les choix narratologiques puisque le narrateur intradiégétique du récit qui alterne entre moments d'actions et introspections s'exprime à travers la voix off dans le film. Sur ce point, la figure de Randal créée par Louis Malle entre parfaitement en résonance avec celle de Darien. Alors qu'André Breton ([1955] 1996 : 103-107) considère Le Voleur comme «le plus rigoureux assaut [qu'il] sache contre l'hypocrisie, l'imposture, la sottise, la lâcheté », le cinéma accentue cet aspect déjà omniprésent dans le roman en représentant une sorte de dandy vengeur bien loin du gentleman cambrioleur à la Lupin. L'accomplissement de Randal se fait dans la marge, et le film reste fidèle à une éthique subversive déjà fortement marquée dans la table du Voleur où le lexique de l'amoralité est récurrent (Darien [1897] 1994 : 525-526) : « voleurs »; « le vice »; « machiavéliques ». Avec de tels choix lexicaux, l'idée d'un vol avec effraction et sans scrupule se justifie avant même de lire le récit.

Dès les premières images en voix off, le film acte cette orientation en lui ajoutant une dose de fatalisme :

Il y a des voleurs qui prennent mille précautions pour ne pas abîmer les meubles. Moi pas. Il y en a d'autres qui remettent tout en ordre après leur visite. Moi jamais. Je fais un sale métier mais j'ai une excuse, je le fais salement. [...] Cambrioler c'est ma vie. On ne choisit pas sa destinée, mais je ne me plains pas.

Le réalisateur aménage - en le développant davantage - un passage se trouvant au chapitre $\mathrm{x}$ du roman : «Les voyages forment la jeunesse ». De la sorte, en nous situant au cœur de l'effraction par un discours intérieur et un univers sonore du bris (verre) et de la fracture (meubles), Louis Malle campe le décor d'un film dont le séquençage est tout entier organisé autour de cette scène initiale. C'est d'autant plus vrai que ce cambriolage sert aussi de clôture au film lorsque Randal - incarné par un Jean-Paul 
Belmondo sans état d'âme - quitte ces mêmes lieux sans être inquiété. Dans une atmosphère Belle Époque, l'acteur incarnant le voleur ne fait qu'appliquer concrètement une reprise individuelle chère à l'auteur du Voleur, laquelle reflète une sensibilité anarchiste visant non pas tant à voler mais à agresser, faisant « de l'offense au coffre-fort le symbole d'une offensive générale contre la forteresse des institutions bourgeoises » (Masson 1994 : 508-509). En procédant de la sorte, tout laisserait à penser que le film s'éloigne du roman-feuilleton.

Il n'en est rien car Louis Malle sait tirer les ficelles d'un genre tout le long du film. Il le fait dès la scène qui pose le cadre et qui pourrait représenter la situation initiale et la situation finale d'un récit: c'est d'abord un effet de suspense sur lequel joue le réalisateur à travers le début in medias res. Le spectateur voit Randal en plein vol et identifie une adaptation de la métaphore de l'«aurore", celle qui ouvre le premier chapitre de la fiction de Darien par un titre symbolique. Au cinéma, cet emblème romanesque prend une tout autre valeur dans l'une des dernières scènes du film lorsque dialoguent Randal et Charlotte. Le jeune homme, après avoir assouvi sa vengeance en falsifiant le testament de son oncle Urbain en train d'agoniser, explique à la jeune femme ses motivations en tant que voleur et compare cette existence à une forme de naissance : « La nuit dans une maison inconnue quand tout dort et que j'arrive et qu'il y a cette chose éventrée, c'est comme si je venais au monde."

Outre ce moment qui représente de manière emblématique le vol, le reste du film propose une succession d'aventures qui pourrait aisément se résumer à un titre du Voleur tel que "Rencontres heureuses et malheureuses» au chapitre xIII. Cette intitulation se matérialise au cinéma non pas par un travail des titres, mais plutôt par les choix des enchainements de séquences. Force est de constater que deux approches apparaissent dans la réalisation du film. D'un côté, Louis Malle travaille à de rares occasions - d'où l'importance lorsqu'il le pratique - le séquençage par un fondu blanc. De cette manière, le film marque le passage temporel entre le vol initial de Randal adulte et l'image du héros enfant se rendant sur la tombe de ses parents accompagnés de Charlotte - sa cousine - et de son oncle - à l'origine de son malheur et de son désir de vengeance. Ce fondu comble un écart temporel conséquent que le réalisateur tient de cette façon à souligner. De l'autre côté, le spectateur identifie dans le reste du film un séquençage sans transition aucune. Dans un rythme quasi effractionnaire, on passe allégrement d'une scène de larcin à une scène de mondanités, sans oublier les moments de dialogue à propos d'une société altérée moralement et courant à sa perte en plein décadentisme fin-de-siècle.

Ce sont d'ailleurs ces moments que le film met en relief en faisant alterner actions et raisonnements. De ce point de vue, le grief de Pascal Pia (1955: 307) à l'encontre du Voleur qui aurait voulu pouvoir "faire abstraction des conversations au cours desquelles Randal et ses acolytes commentent leur activité et jugent la société qu'ils mettent en coupe réglée [...] » ne tient pas, car c'est à partir de cet équilibre entre deux pôles que le film parvient justement à sublimer les ressorts narratifs d'une intrigue à la liberté toute picaresque. Ainsi, la réception en demi-teinte du film, au même titre que le roman de Darien, ne doit pas effacer la dynamique qui préside à la réalisation de ce film restant globalement fidèle à quelques grands principes du roman-feuilleton en vogue au XIX ${ }^{e}$ siècle. Entre les intrigues sentimentales (Renée), les rencontres avec des personnages types tels que le curé nihiliste Lamargelle ${ }^{26}$ ou le bagnard évadé Canonnier, les moments de solitude du héros qui songe à ses actes, Le Voleur au cinéma 
ne porte pas atteinte à l'esprit du roman, au contraire. Il approfondit une vision idéologique à l'œuvre dans la fiction d'origine et, de ce point de vue, la démarche éthique de Louis Malle rejoint celle de Georges Darien. Les deux hommes ont en commun le désir de remettre en question leurs milieux d'origine respectifs : le monde des riches industriels pour le premier et celui de l'univers petit-bourgeois des commerçants pour le second. Le Voleur tant dans la fiction romanesque que dans l'adaptation au cinéma sert ce dessein contestataire qui rapproche les deux artistes.

Toutefois, il n'en demeure pas moins que le film reste une adaptation du roman et ne peut pas tenir compte de l'ensemble des éléments de l'intrigue darienienne. La multiplication des rebondissements n'est pas traitée au cinéma. Pour autant, les recours aux analepses - retours sur l'enfance dans le roman - comme la teneur de discours tels ceux de l'abbé prévaricateur incarné par l'abbé Lamargelle - toujours dans les sous-sols en train de fomenter ses prochains larcins comme un religieux satanique montrent une reprise attentive de l'ordonnancement du roman et de ses étapes annoncées dans les titres de chapitres.

Outre cet aspect, il faut tout de même noter que, pour des raisons de réalisation, le film gomme, en partie, les motivations sociales et politiques du héros. Des passages comme la rencontre d'Annie la pauvresse, scène pleine de pathos et de réflexion sur les jugements arbitraires d'une société, n'apparaissent pas dans le film. Nonobstant, le réalisateur garde certains épisodes qui rappellent l'un des cadres idéologiques fondamentaux du roman-feuilleton: le mélodrame ${ }^{27}$. Ce genre théâtral repose, entre autres, sur un principe moral: la lutte entre le Bien et le Mal. Au cinéma, cette dimension s'impose par une succession d'aventures où le jugement éthique repose sur une symbolique autour du vice et de la vertu. De la sorte, Louis Malle oriente, dans une certaine mesure, Le Voleur vers la forme argumentative de l'apologue puisque les premières paroles du film sont d'ordre moral, au même titre que les ultimes échanges à la fin lorsque le spectateur revient à la scène d'ouverture : le cambriolage. En cela, le film est semblable au roman car tous deux mettent en évidence cette introspection du héros face à ses actes, lesquels sont l'occasion de revenir sur les origines d'une révolte enfantée par la société de la petite bourgeoisie qui a bridé le jeune individu. En retour, ce dernier exerce une reprise individuelle jugée par ses soins nécessaire, voire salutaire. Ce lien qui unit les destins des héros du roman et du film est d'autant plus frappant que le geste ultime accompli par Belmondo dans le film de Malle fait écho à celui réalisé par Randal au sortir du roman : un abandon symbolique de leurs outillages de voleur.

De l'œuvre littéraire au cinéma, le processus de transécriture pose les bases d'une effraction générique qui n'en est pas vraiment une, puisque Louis Malle reste plutôt fidèle au romanesque subversif auquel tient Darien dès l'épigraphe de son récit phare signé La Fontaine: "Les voleurs ne sont pas / Gens honteux ni forts délicats.» Toutefois, il opère un certain nombre d'adaptations en partie liées à un cadre générique autrement contraignant puisque les techniques narratives et les techniques cinématographiques enrichissent de manières parfois différentes le rapport au Voleur et à l'influence du roman-feuilleton. Il en est de même concernant une autre adaptation de l'œuvre de Darien, cette fois via le médium bédéique. 


\section{Du septième art à la bande dessinée : le roman- feuilleton entre cadre et marge idéologiques} n'est plus question de titres ou de séquences, mais bien de planches. Ces dernières mettent en évidence une manière de concevoir autrement la lecture du roman de Darien. La construction générale du texte repose sur quelques éléments récurrents : organisation autour de lieux emblématiques comme Malenvers, Bruxelles, Londres, Paris, et de figures incontournables telles que Lamargelle ou Paternoster. Bernard Seyer reprend donc des éléments centraux de l'intrigue de 1897 et insiste sur le cosmopolitisme du monde des voleurs, celui que Darien évoque dans l'avant-propos de son roman écrit à Londres en 1896. Cette composante aurait pu, à bien des égards, nourrir un esprit feuilletonesque dans la bande dessinée.

Ce n'est pas vraiment le cas car, à l'intérieur même de la fiction, le bédéiste effectue des choix en mettant notamment de côté le héros inspiré de Jules Mary et du romanfeuilleton de type social, Roger la Honte. Dès lors, l'esprit même du vol et de la reprise individuelle omniprésent dans la fiction fin-de-siècle cède la place à une autre manière de concevoir l'approche du texte de Darien. Même si l'intertexte du feuilleton est absent en tant que tel, il n'en demeure pas moins que les planches mettent en évidence des éléments représentatifs de l'esprit du genre : intrigues sentimentales en série avec Charlotte et au cours de la scène du bal qui donne lieu à un crime d'adultère dans le chapitre XXIII du Voleur : «Barbe-bleue et le domino noir ». À travers ces événements, la bande dessinée met en lumière une tonalité tragique plus remarquable qu'elle ne l'est dans l'œuvre originelle.

Cette interprétation est d'autant plus avérée si l'on considère les choix narratologiques qui sont ceux de Seyer : univers en noir et blanc qui met au jour l'importance accordée aux gros plans sur les visages et donc, indirectement, les consciences duelles tiraillées entre le Bien et le Mal - influence du mélodrame; le poids d'une bourgeoisie et l'envie de s'en affranchir. Sur ce dernier point, les formules clés tirées du roman donnent justement à l'ouvrage en question une valeur fortement symbolique car le poids des mots accompagne les impressions laissées par les dessins dans les différentes vignettes. Ainsi, la première planche s'ouvre sur une formule du héros prononcée dans Le Voleur au chapitre xxx, ultime propos avant d'abandonner là son équipement de voleur : " Dire qu'on est toujours volé par quelqu'un... Ah ! chienne de vie $! . . .{ }^{28}$ » (Seyer 1986: 9). Ces mots sont en corrélation avec les premières images qui montrent justement un personnage amer rendant compte de sa jeunesse volée. On plonge alors dans l'univers $\mathrm{du}$ cambrioleur sous couvert d'une idéologie bourgeoise incarnée par l'oncle Urbain Randal, le même qui est à l'œuvre dans le roman. L'image en fait un stéréotype qui conforte l'esprit bourgeois par une apparence ventripotente et cynique. De la sorte, la bande dessinée offre un régime visuel que Darien - il faut le souligner - a mis de côté, car il rejette le principe de la description dans les romans de mœurs réalistes et naturalistes de l'époque ${ }^{29}$. Sur la dernière planche, l'esprit reste le même en conjuguant à la fois la restitution des pensées de Randal et l'image programmatique. Le refus de conclure en évoquant des "dénouements de théâtre " et des "épilogues de fiction » conduit le personnage à affirmer que "ça ne finit jamais: ça continue...» (54). L'aposiopèse s'accompagne d'une mention faite au voyage par la représentation d'un port et de bateaux. Le regard de Randal en arrière-plan fait une dernière fois écho à 
l'entreprise romanesque autour d'un narrateur intra-diégétique, lequel laisse entrevoir le flux de sa conscience meurtrie et révoltée.

L'ensemble de la bande dessinée repose d'ailleurs sur la centralité des regards. La plupart des planches montrent essentiellement, outre des plans généraux qui se focalisent sur des espaces urbains pour la plupart, des plans rapprochés et des gros plans $^{30}$. Dès lors, la bande dessinée respecte une certaine manière de concevoir le parcours du héros romanesque, à savoir un protagoniste dont les tourments intérieurs - familiaux entre autres - le conduisent à porter un jugement sur la société fin-desiècle, lequel passe ensuite par l'exercice d'un «métier» (Darien [1897] 1994 : 203), celui de voleur.

Cette mise en œuvre pratique de l'activité en question passe dans la bande dessinée par un ensemble d'éléments qui reprennent, dans une certaine mesure, les aspects les plus saillants du roman-feuilleton. Sans pouvoir œuvrer dans la logique du chapitre court et des enchaînements romanesques, le bédéiste parvient tout de même à rendre compte des aventures les plus représentatives de la veine du feuilleton qui crée chez le lecteur, au tournant du siècle, une attente sans cesse renouvelée par les coups de théâtre et les intrigues de la sentimentalité et des affaires. Le lecteur redécouvre ainsi, non pas tant le pastiche, mais bien l'adaptation de scènes comme les amours contrariées de Georges et Charlotte, lesquels se retrouvent autour de leur fille Hélène qui souffre d'une méningite. La course contre la montre et contre la mort conduit à une intrigue policière autour du crime et de la culpabilité, ce qui n'est pas sans rappeler le romanfeuilleton qui exploite fréquemment les récits criminels, lesquels sont à la fois un moteur narratif et un motif qui tient en haleine le lecteur. D'abord topos romantique qui se perpétue chez Victor Hugo dans Les Misérables et dans certains romans d'aventures ou policiers avec une figure telle qu'Arsène Lupin, le gentlemancambrioleur, l'intrigue criminelle trouve naturellement une place de choix dans le roman-feuilleton qui dépeint les bas-fonds et les vicissitudes de l'existence de héros stéréotypés (Eugène Sue). Dès lors, la délinquance offre un potentiel romanesque et bédéique indéniable que les auteurs exploitent à leur manière en opposant symboliquement le principe de la récupération individuelle - vol jugé légitime - à la spoliation volontaire par les tenants de l'autorité bourgeoise, lesquels sont maître d'un pouvoir qu'ils construisent contre l'individu. Cette confrontation de deux mondes permet, au fond, de rendre palpable pour le lecteur un suspens narratif à haute teneur mélodramatique. Cette sensation est particulièrement visible dans la vignette de la bande dessinée concernant justement l'épisode de la mort de Paternoster, événement vecteur de romanesque qui prend ici une dimension visuelle accentuant le pathétique mélodramatique déjà perceptible dans les mots du narrateur Randal: «Et si Paternoster me refusait ce que je veux lui demander? S'il ne voulait rien entendre? ... » (Seyer 1986 : 38). En arrière-plan de la scène (38), on voit, de fait, un gros plan sur le visage inquiétant de Paternoster le faussaire ainsi que le mot «Murdock» en lettres capitales sur une enseigne commerciale ${ }^{31}$. Les planches qui suivent jouent sur ces visages crispés, souffrants, aussi bien du côté de l'homme que de l'enfant malade. Il en est de même dans l'intrigue du bal à Paris qui s'achève sur une planche où l'expressionnisme est des plus tragiques. Les visages se tordent comme dans une vision fantastique donnant à l'apologue servant d'intertexte au roman - «Barbe-bleue », une autre tonalité typiquement feuilletonesque, un peu à la manière des séries de mystères qui marquent le XIX ${ }^{e}$ siècle littéraire. Ainsi, la valeur programmatique du titre de Darien 
permet dans la bande dessinée de déployer un imaginaire visuel fort, amplifiant l'effet coup de théâtre récurrent dans les feuilletons.

Cette influence est d'autant plus puissante que le principe même de la bande dessinée oblige à concentrer les effets comme a dû le faire Darien dans les courts chapitres qui composent son Voleur. En l'adaptant dans une forme générique possédant ses propres contraintes, le bédéiste a mis en valeur la dualité action et discours en choisissant les événements les plus susceptibles de représenter l'enjeu idéologique général du récit darienien. Même si la dimension politique et sociale est mise en retrait, l'intrigue garde en définitive son sel par l'organisation efficace d'un foisonnement picaresque déjà remarquable dans le texte de 1897. Cette dimension picaresque prend une tournure mélodramatique dans le roman et dans la bande dessinée car les deux œuvres polarisent des attitudes - hypocrisie des uns et sincérité des autres - et jouent le mélange des tonalités à travers une série de péripéties où l'idée de justice comme d'injustice est au cœur des enjeux narratifs. D'une certaine façon, les deux écritures du Voleur réinvestissent une rhétorique de la surenchère qui passe d'un côté par les mots et de l'autre davantage par le régime visuel qui guide le lecteur vers une autre façon de lire et d'imaginer la réalité du Voleur.

Dans le cadre de genres hybrides tels que le roman-feuilleton, pris entre romanesque et mélodrame, la réécriture apparaît comme un exercice complexe auquel Darien s'est astreint dans Le Voleur en 1897. L'intrigue de l'œuvre, dès l'intitulation, reprend un code essentiel des romans parus quotidiennement dans la presse au $\mathrm{XIX}^{\mathrm{e}}$ siècle: la périodicité du journal pour le feuilleton. Seulement, loin de simplement reproduire les schémas narratifs d'une mode littéraire qui plaît au public, Darien pastiche le romanfeuilleton en puisant à la source de ses influences nombreuses (roman populaire, roman criminel, roman horrifiant, trames mélodramatiques en vogue) et parfois même semble parodier les modèles dont il s'inspire fortement. Ainsi, le romancier relâche-t-il le lien sérieux qui unit son récit à d'autres formes génériques du romanesque pour proposer à ses lecteurs une fiction, in fine, seuil, c'est-à-dire à la frontière du pastiche, de la parodie et à la croisée de catégories romanesques nombreuses. Cette stratégie ne répond-elle pas à l'une des volontés de Darien qui est de ne se pas compromettre dans l'écriture de romans : «Le roman, voyez-vous, c'est encore ce qui nous divise le moins [...]. Puis, ce n'est pas trop compromettant, et je ne tiens pas à me compromettre; car se compromettre, c'est se donner, et je ne veux pas me donner» (Stock 1935: 73). Ce refus de se rendre lisible - en termes de stratégie auctoriale - pour ses lecteurs justifie, sans aucun doute, cette plasticité facétieuse avec laquelle Darien manie un art romanesque du modèle et de l'adaptation de ce dernier. Cette hybridité est aussi valable dans les adaptations contemporaines du Voleur qui traitent de la narration darienienne avec les déterminations de supports médiatiques permettant de (re)lire le roman : l'enchaînement des scènes pour le film et la séquentialité iconotextuelle pour la bande dessinée. 


\section{BIBLIOGRAPHIE}

Angenot, Marc, 1989, 1889. Un état du discours social, Montréal, Le Préambule.

Aron, Philippe, 2008, Histoire du pastiche, Paris, PUF.

Artiaga, Loïc (dir.), 2008, Le roman populaire en France (1836-1960). Des premiers feuilletons aux adaptations télévisuelles, Paris, Éditions Autrement.

Aubry, Danielle, 2006, Du roman-feuilleton à la série télévisée. Pour une rhétorique du genre et de la sérialité, Berne, Peter Lang.

Auriant, 1955, Darien et l'Inhumaine comédie, Bruxelles, Ambassade du Livre.

Baroni, Raphaël, 2007, La Tension narrative. Suspense, curiosité, surprise, Paris, Seuil.

Bosc, David, 1996, Georges Darien, Aix-en-Provence, Sulliver.

Breton, André, [1955] 1996, « Darien le maudit », Perspective cavalière, Paris, Gallimard, p. 103-107.

Compère, Daniel, 2012, Les romans populaires, Paris, Presses Sorbonne Nouvelle, coll. « Les

fondamentaux de la Sorbonne nouvelle ».

Darien, Georges, 1906, « Incident Bernstein-Darien », Le Figaro, nos 244, 247, 258, p. 4.

Darien, Georges, [1897] 1994, Le Voleur, Paris, Seuil, coll. « L'école des lettres ».

Darien, Georges, [1890] 2002, Biribi, Paris, Le Serpent à Plumes, coll. « motifs ».

Deprêtre, Évelyne et Duarte, German A. (dir.), 2019, Transmédialité, bande dessinée et adaptation, Clermont-Ferrand, Presses Universitaires Blaise Pascal.

Genette, Gérard, 1972, Figures III, Paris, Seuil.

Genette, Gérard, 1992, Palimpsestes. La littérature au second degré, Paris, Seuil.

Gréau, Valia, 2002, Georges Darien et l'anarchisme littéraire, Tusson, Du Lérot.

Horvath, Christina, 2008, Le roman urbain contemporain en France, Paris, Presses de la Sorbonne Nouvelle.

Kalifa, Dominique, 2005, Crime et culture au XIX ${ }^{e}$ siècle, Paris, Perrin.

Kalifa, Dominique et Thérenty, Marie-Ève (dir.), 2015, Les Mystères urbains au XIX siècle: Circulations, transferts, appropriations, Médias19, [En ligne], http://www.medias19.org/index.php? id $=17039$

Malle, Louis, 1967, Le Voleur, film adapté du roman de Georges Darien, NEF, Artistes associés, 120 minutes.

Mary, Jules, 1887-1889, Roger-la-Honte, Paris, Jules Rouff.

Masson, Pierre, 1994, postface au roman Le Voleur, Paris, Seuil, coll. « L'école des lettres », p. 507-520.

Musset, Alfred, [1831] 1863, « La Coupe et les lèvres », Premières poésies, Paris, Charpentier.

Pezard, Émile, 2013, « La Vogue romantique de l'horreur : roman noir et genre frénétique », Romantisme, $\mathrm{n}^{\circ} 160$, p. 41-51.

Pia, Pascal, 1955, « Georges Darien et Le Voleur », Les Lettres Nouvelles, n 301, p. 300-312. 
Queffélec, Lise, 1989, Le roman-feuilleton français au XIX ${ }^{e}$ siècle, Paris, PUF, coll. « Que sais-je ? ».

Reboul, Olivier, 1980, Langage et idéologie, Paris, PUF.

Rico, Francisco, 2000, La novela picaresca y el punto de vista, Barcelone, Seix Barral.

Seyer, Bernard, 1986, Le Voleur, Paris, Les Humanoïdes associées, coll. « Métal Hurlant ».

Stock, Pierre-Victor, 1935, « Georges Darien », Mémorandum d'un éditeur, Paris, Stock, p. 59-102.

Terrone, Patrice, 2000, « Sataniques, mon père... », Recherches et Travaux, nº 50, p. 121-139.

Thiesse, Anne-Marie, 2000, Le Roman du quotidien. Lecteurs et lectures populaires à la Belle Époque, Paris, Seuil, coll. « Points ».

\section{NOTES}

1. Feuilleton renvoie, en effet, au bas de la page d'un journal où étaient publiés ces romans afin d'être éventuellement découpés et reliés.

2. Pour cerner cette catégorie littéraire, voir les travaux de Loïc Artiaga (dir.), Le roman populaire en France (1836-1960). Des premiers feuilletons aux adaptations télévisuelles (2008) et Daniel Compère, Les romans populaires (2012).

3. Héros de Ponson du Terrail dans le roman de 1857 intitulé Les Drames de Paris. Le héros en question incarne, après bien des mésaventures (usurpation d'identité, vol, chantage, crime), le redresseur de torts en marge de la société.

4. Personnage emblématique de Jules Mary en 1886.

5. Pour une définition du pastiche et de son lien complexe avec la parodie, voir Philippe Aron, Histoire du pastiche (2008).

6. La formule est reprise à Raphaël Baroni dans La Tension narrative. Suspense, curiosité, surprise (2007).

7. Pour cette articulation, voir l'étude de Dominique Kalifa et Marie-Ève Thérenty (dir. 2015).

8. Le mot renvoie ici à la notion forgée par Gérard Genette pour désigner le paratexte.

9. Voir Danielle Aubry (2006).

10. Cette fois le mot seuil renvoie de manière étendue à des questions telles que le seuil entre le roman et ce qui ne l'est pas, entre le récit et ce qui s'apparente à un exposé idéologique, entre le roman populaire et d'autres approches romanesques dont l'influence peut se faire ressentir à la lecture du Voleur. L'approche de la notion de «fiction seuil» se veut donc multidirectionnelle afin d'envisager au mieux la nature plurielle du texte de 1897.

11. Pour cette notion de «transmédialité », voir Évelyne Deprêtre et German A. Duarte (2019).

12. Les termes renvoient aux critères de l'idéologie établis par Olivier Reboul (1980: 21-25).

13. Nous reprenons l'expression à Dominique Kalifa qui analyse la littérature criminelle du XIX siècle et dépasse les problématiques liées aux genres que pose le feuilleton: "Utilisée par les contemporains, l'expression "roman criminel", qui regroupe à la fois les grands cycles feuilletonesques du milieu du siècle (Sue, Dumas, Féval, Ponson du 
Terrail...), le roman judiciaire (Gaboriau et ses suiveurs) et les prémices du roman de détection, permet d'éviter les querelles génériques sur la "naissance" du roman policier, et met l'accent sur la spécificité alors reconnue à ces textes: des récits d'aventures où la rupture criminelle donne lieu à une exploration plus ou moins méthodique du monde social» (Kalifa 2005 : 144). Le Voleur de Darien explore justement le monde social fin-de-siècle qui caractérise les grandes villes telles que Paris, Londres et Bruxelles, lieux emblématiques par lesquels passent les héros du roman. Au cœur de ces espaces, le crime et la corruption s'exercent dans le seul but de pratiquer une reprise individuelle indispensable pour l'individu opprimé par les autorités de tous ordres.

14. Voir Émile Pezard (2013).

15. Marc Angenot (1989) part du principe que dans la seconde moitié du XIX siècle, en France, la gnoséologie en vigueur est « romanesque ». Toutes les caractéristiques d'un «romanesque général» sont réunies pour faire du roman un modèle de toutes les pratiques discursives de l'époque dans leur versant gnoséologique.

16. Cette pièce n'a jamais été publiée ou représentée. Dès lors, s'agissait-il d'une autre facétie de l'écrivain contestataire?

17. Notons que les origines du roman picaresque remontent $a u \mathrm{XVI}^{\mathrm{e}}$ siècle espagnol avec Lazarillo de Tormes notamment. Pour approfondir la réflexion sur le roman picaresque, on peut se reporter aux travaux de Francisco Rico (2000).

18. Terme employé par David Bosc (1996 : 73).

19. Titre renvoyant à un poème de Musset ([1836] $1863: 252)$.

20. L'expression ne doit pas être confondue avec le genre du roman noir qui apparaît aux États-Unis dans les années 1920-1930 et qui intègre, à la différence du roman policier, une critique sociale. Dans notre emploi, l'expression renvoie à ces productions romanesques de la noirceur au temps du romantisme (Mérimée, Gautier, etc.). Dès lors, ce roman noir se caractérise par la filiation avec des modèles et la création de lignées génériques telles que le gothique (veine anglo-saxonne), le romantisme frénétique (Nodier, entre autres), les interactions avec le mélodrame, ce qui a eu indéniablement, à divers degrés, des influences sur le roman-feuilleton romantique à la croisée des genres. De tels lignages, complexifient l'approche théorique de ce phénomène littéraire $\mathrm{du}$ romantisme noir au XIX ${ }^{\mathrm{e}}$ siècle.

21. L'expression n'est pas à confondre avec la catégorie romanesque du roman sentimental. Ce genre de romans se développe dans la presse et l'édition catholique vers la fin du XIX ${ }^{\mathrm{e}}$ siècle. Signés Zénaïde Fleuriot, Marie Maréchal ou Mathilde Bourdon dans les périodiques L'Ouvrier (1861-1919) et Les Veillées des chaumières (à partir de 1877), ces romans sentimentaux ne sont pas à l'honneur au temps du romantisme noir et criminel $\mathrm{du}$ roman-feuilleton. Présents quasi systématiquement, mais pas centraux dans l'intrigue, ces sentiments justifient l'appellation choisie dans le corps de l'article.

22. Voir l'étude d'Anne-Marie Thiesse, Le Roman du quotidien. Lecteurs et lectures populaires à la Belle Époque (2000). Un roman-feuilleton comme La Porteuse de pain centre l'action non plus sur le héros qui mène l'action, mais sur la victime qui la subit, laquelle est en proie à bien des sentiments.

23. Traduit de l'anglais ce mot veut dire le scélérat. Dès lors, tout semble conduire au crime et aux tourments intérieurs qui frappent le voleur Randal devenu criminel alors qu'il s'y est toujours refusé. 
24. Personnage principal du roman Biribi.

25. Certains extraits du film feront l'objet d'une retranscription dans le corps de l'article.

26. L'ambivalence de ce curé dont les incursions surprises dans le récit sont fondamentales pour le héros individualiste Randal, Patrice Terrone (2000: 125) la souligne en ces termes: "[...] il appartient au siècle en même temps qu'il professe l'éternité, simple mortel appelé à diffuser la parole divine, reproducteur par ses actes des grands mystères de l'eucharistie, de la rémission des péchés, le prêtre incarne pourrait-on dire l'inconciliable : l'humain trop humain, soumis aux tentations de la chair, de la possession, de l'argent, du confort, du pouvoir, et la sainteté à laquelle il doit aspirer ». Cette dualité du prêtre intéresse le réalisateur Louis Malle qui rend cette figure incontournable dans l'adaptation filmique du Voleur.

27. Illustré notamment par Alexandre Dumas avec Les Trois mousquetaires et La Dame de Montsoreau. En effet, les textes de Dumas comportent une trame mélodramatique car ils reprennent à la fois des motifs (amours empêchées, triomphe de la vertu, châtiment exemplaire du crime) et des personnages types (le traître, l'héroïne). Ces ingrédients qui plaisent à un public populaire désireux d'assister à la vertu victorieuse, le tout à travers les sentiments et les rebondissements, voilà ce qui nourrit, en partie, le romanfeuilleton.

28. Pour rappel, cette formulation est textuellement reprise à la conclusion provisoire du Voleur de Darien. Voir supra.

29. Ce refus de la description est un motif récurrent dans les œuvres de fiction. On peut citer parmi les déclarations de Darien celle tirée du Voleur lorsqu'une jeune femme demande au narrateur Georges Randal de lui décrire la ville de Malenvers. C'est l'occasion pour l'écrivain de rappeler son mépris du naturalisme descriptif : «Si vous voulez le savoir, faites comme moi ; allez-y. Ou bien, lisez un roman naturaliste ; vous êtes sûrs d'y trouver quinze pages à la file qui peuvent s'appliquer à Malenvers. Moi, je ne fais pas de descriptions; je ne sais pas. Si j'avais su faire les descriptions, je ne me serais pas mis voleur » (Darien [1897] 1994 : 256).

30. Le cas du plan moyen permet, en revanche, d'accentuer le lieu commun autour de figures emblématiques comme le bourgeois incarné, entre autres, par l'oncle Urbain.

31. Ladite enseigne pouvant jouer par paronymie sur le mot anglais "murder", ce dernier convoquant le motif emblématique du crime, élément central de cette scène.

\section{RÉSUMÉS}

Le Voleur de Georges Darien est un roman qui relate les aventures de Georges Randal. Ce jeune homme œuvre en faveur d'une reprise individuelle qui trouve son origine dans l'injustice familiale incarnée par un oncle fourbe et cupide. À partir de là, le récit se construit comme un pastiche de roman-feuilleton, lequel s'envisage à la fois dans sa chronologie - périodisation du genre - et dans ses principes logiques - critères définitoires. Ce caractère générique, outre Darien qui vraisemblablement a cherché à écrire une suite aux mésaventures de son héros au début du 
$\mathrm{xx}^{\mathrm{e}}$ siècle, d'aucuns s'en inspirent dans les adaptations connues du Voleur. Du cinéma avec le film de Louis Malle en 1967 à la bande dessinée de Bernard Seyer parue aux Humanoïdes associés en 1986, le feuilleton se réactualise sans dénaturer la dynamique romanesque de l'intitulation et du contenu narratif défendue par Darien dans ce qui s'apparente en 1897 à un antiroman écrit par celui qui aspire à être le romancier contestataire de son époque fin-de-siècle.

The Thief, by George Darien, is a novel telling Georges Randal's adventures. This young man strives to recover himself, because of an injustice, rooted in his family by a false-heathed and grasping uncle. Then the story becomes a pastiche of serialized novel, at once in the stages of chronology, and in its defining criterions. Darien uses this characteristic of genre, probably hoping to write following episodes of his heroe's misadventures, at the beginning of the twentieth century. And there are some who are inspired by this model, adapting The Thief: in 1967, Louis Malle produces a film, and a comic book by Bernard Seyer is published in 1986 by Humanoïdes associés. So the serial is brought up to date, without distorting the novelistic dynamics of entitling and of narrative content stood by Darien. And this novel (1897) has certain similarities to an antinovel, written by the man who longed for being the anti-authority novelist in the fin de siècle period.

\section{INDEX}

Mots-clés : Darien (Georges), Voleur (Le), pastiche, roman-feuilleton, Malle (Louis), bande dessinée, cinéma, chapitre

Keywords : Darien (Georges), Thief (The), pastiche, serialized novel, Malle (Louis), comic books, cinema, chapter

\section{AUTEUR}

\section{AURÉLIEN LORIG}

Université Sorbonne Nouvelle, CRP19 